\title{
RBF Neural Network Control for Linear Motor-Direct Drive Actuator Based on an Extended State Observer
}

\author{
Zhi Liu and Tefang Chen \\ School of Traffic and Transportation Engineering, Central South University, Changsha, China \\ Correspondence should be addressed to Zhi Liu; 124201002@csu.edu.cn
}

Received 27 June 2016; Accepted 16 October 2016

Academic Editor: Juan R. Torregrosa

Copyright (c) 2016 Z. Liu and T. Chen. This is an open access article distributed under the Creative Commons Attribution License, which permits unrestricted use, distribution, and reproduction in any medium, provided the original work is properly cited.

Hydraulic power and other kinds of disturbance in a linear motor-direct drive actuator (LM-DDA) have a great impact on the performance of the system. A mathematical model of the LM-DDA system is established and a double-loop control system is presented. An extended state observer (ESO) with switched gain was utilized to estimate the influence of the hydraulic power and other load disturbances. Meanwhile, Radial Basis Function (RBF) neural network was utilized to optimize the parameters in this intelligent controller. The results of the dynamic tests demonstrate the performance with rapid response and improved accuracy could be attained by the proposed control scheme.

\section{Introduction}

Currently, the most common electrohydraulic actuator is the multistage hydraulic actuator with the nozzle flapper pilot [1]. But this kind of actuator has disadvantages such as poor anticontamination characteristics, low-frequency response, and low-power density. So, the linear motor-direct drive actuator (LM-DDA) system [2, 3], which would greatly simplify the mechanical structure and improve the control frequency response, has attracted great attention in recent years. Compared to the traditional hydraulic actuator, the largest difference is the abolition of the amplification stage [4]. In the LM-DDA system, the spool valve body is straightly driven by the linear motor. So, the frequency of the spool movement has been significantly improved. However, due to the cancellation of the hydraulic amplifier stage, the whole system has strong nonlinearity and uncertainty [5]. The fluid power and other loads directly couple to the linear motor, so fluid power would have a great impact on the system stability and dynamic control performance.

In the position-closed-loop control system of the DDA, the traditional design is established on the hypothesis that the load force disturbance is 0 or a fixed value. The transfer functions of the actual value and the reference value between spool positions are obtained from this method to optimize the closed-loop transfer function for the purpose of accomplishing the controller design. Negative feedback on load force disturbance has a certain extent to attenuate the hydraulic disturbance, but it does not completely eliminate this influence $[6,7]$.

To achieve rapid response and robustness motions, a high-performance controller with advanced control scheme is required to be designed. PID control is the most conventional control method $[8,9]$. In general, the control of the LM is wished to have accurate signal tracking for the command despite the influence of the transient and steady flow force or the fluctuation of the system's mechanical characteristics. In addition, the response should be rapid due to the design requirement. However, the classic linear PID controller has an unavoidable long recovery process and an inevitable error when the system reaches a stable state $[10,11]$. In this case, the classic PID control strategy could not get fast and accurate position response. So, many design techniques have been proposed to tackle this problem. The literature [12] reported a control scheme based on disturbance observer to realize motion control with high accuracy. Yet, this strategy uses low-pass filter so the performance of the actuator would be influenced while tracking the high-frequency signal. Fuzzy control [13] is an intelligent control method on the basis of fuzzy set theory, fuzzy language variables, and fuzzy logic 
reasoning. The basic idea is to experience expert control of the controlled object into a series of control rules. Fuzzy control does not rely on the specific mathematical model of the plant so its applicable scope is wide and it is easy to be achieved by using digital controller when the nonlinear time varying load has certain robustness. Although fuzzy control strategies improve the intelligence, they simply use more control rules. In this case, much expert experience is needed to settle the rules. So, the control accuracy is relatively low without the expert experience.

Artificial neural network $[14,15]$ has the advantages of distributed storage, parallel processing, nonlinear approximation, and self-learning. So, it shows a broad application prospect in the field of linear servo control. ESO is borrowed from the idea of state observer [16], and it would be able to affect the output of the controlled object disturbance expansion into a new state variable. The establishment of state variables can be observed with the expansion of the observer with a special feedback mechanism. ESO disturbance generated does not depend on the specific model and does not need to directly measure the role of disturbances. The algorithm could be written as a discrete form, and it also has the advantages of having a simple structure, having a small amount of calculation, being easy to implement, and being a real-time digital controller. Therefore, ESO is a more versatile and practical disturbance observer [17].

The remainder of this paper is organized as follows. In Section 2, the model of a DDA system is established and how hydraulic power has a bad effect on the control of LM is analyzed. Section 3 introduces ESO into the control system. The optimal parameters in position controller are obtained by minimizing the energy gain between the spool position and the modeling error. And the method is implemented by an improved back-propagation algorithm with the weight balance. Research experiment process is presented in Section 4, where a novel hardware structure of LM-DDA control based on digital signal processor (DSP) and field programmable gate array (FPGA) is given.

\section{Modeling of Direct Drive Actuator System with Linear Motor}

Figure 1 shows the structure of a DDA system.

As is shown in Figure 1, in the LM-DDA system, the linear motor directly connects to the spool. $T$ is the return port, $A$ and $B$ denote the two direction control ports, and $P$ represents the entrance port. The intelligent controller of the spool receives position reference and feedback. And it would generate the current command to drive the linear motor and complete the closed-loop control. The force withstood by the spool includes thrust force, steady-state fluid power, and flow fluid power. Since the body of the actuator is well lubricated, the impact of the friction is ignored.

The dynamic model of spool could be constructed as

$$
m_{s} \frac{d x_{s}^{2}}{d t^{2}}+B_{s} \frac{d x_{v}}{d t}+F_{l}=F_{s},
$$

where $m_{s}$ represents the quality of the spool, $x_{s}$ is the displacement of the spool which could be measured by a displacement sensor, $B_{s}$ is the viscous damping coefficient of the spool, and $F_{l}$ denotes the disturbance of the spool which includes the transient hydraulic force and steady hydraulic force.

With Laplace transformation to (1), it can be drawn that

$$
x_{s}(s)=\frac{1}{m_{s} s^{2}+B_{s} s}\left(F_{s}(s)-F_{l}(s)\right) .
$$

The hydraulic force has great influence on the opening of the spool and would directly affect the flow of the valve and it should be considered. As is mentioned above, the hydraulic force includes the transient hydraulic force and steady hydraulic force. In general, the steady hydraulic force plays a main role. The existence of the steady hydraulic force increases the driving force of the spool and could cause vibration and nonlinear problem, yet there is no mechanical compensation or pressure drop which would completely eliminate the influence of the steady axial hydraulic force.

The steady hydraulic force could be expressed as follows:

$$
F_{\mathrm{st}}=-2 C_{V} \omega X_{v} \Delta p \cos \theta
$$

The transient hydraulic force is

$$
F_{\mathrm{tr}}=\rho\left(L_{1}-L_{2}\right) C_{V} \omega \sqrt{\frac{2}{\rho} \Delta p} \frac{d X_{v}}{d t}
$$

where $X_{v}$ is the area of discharge, $\Delta p$ exemplifies the pressure which drops across the outlet, $\omega$ is the region gradient of the DDA, $\left(L_{1}-L_{2}\right)$ is the length of the damper, $C_{V}$ represents the coefficient of the volume, $\theta$ denotes the angle of the flow, and $\rho$ is the density of the oil liquid.

The existence of the axial hydraulic power increases the driving force of the spool, and it would cause the vibration and nonlinear problem. Due to the fact that the axial hydraulic power is related to the operation condition of the DDA, it is difficult to achieve the real-time compensation of fluid dynamics. And this phenomenon could not be completely eliminated. Figure 2 illustrates the whole controlling process of the LM-DDA system. Because the conventional method could not meet the design requirement, the parameters of the position control are first adjusted by the RBF neural network automatically, and then the hydraulic disturbance is estimated by the ESO.

\section{The Proposed Control Scheme}

ESO is borrowed from the ideas of the state observer; it is able to get the new state variables from the disturbance function that would influence the controlled output. Special feedback mechanism is established to observe the extended state variables. The basic mechanism of the ESO is that since the alleged output is observable, the output signal contains the information about the disturbance. So, it could be extracted from the output. In this paper, ESO is used to establish the model of the unknown external disturbance (unpredictable hydraulic force). 


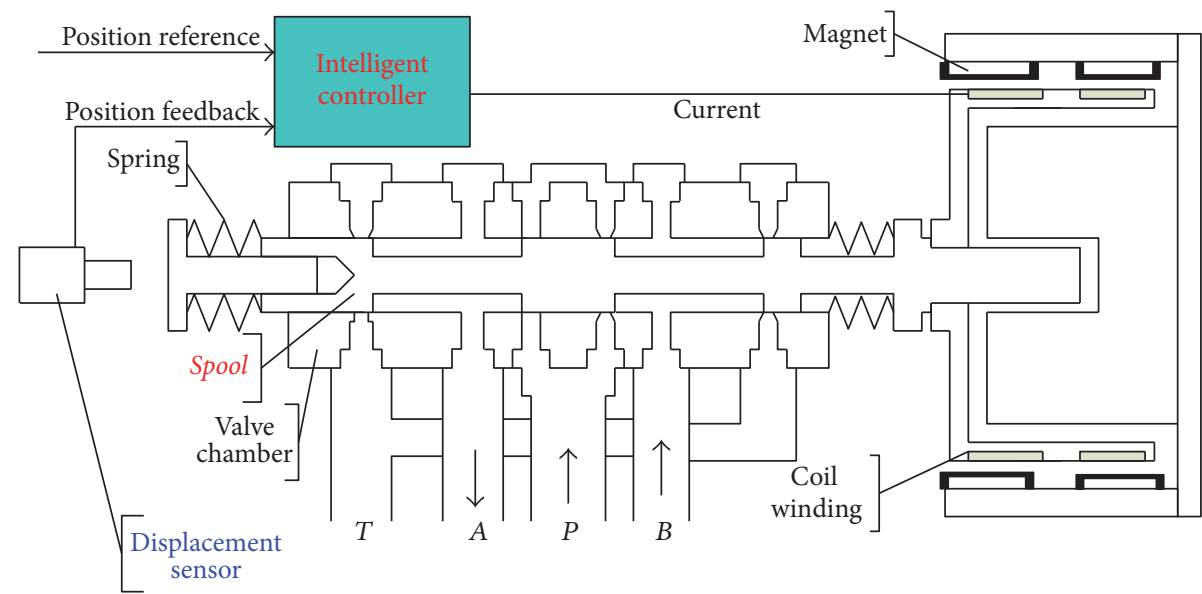

FIgURE 1: The structure of the DDA.

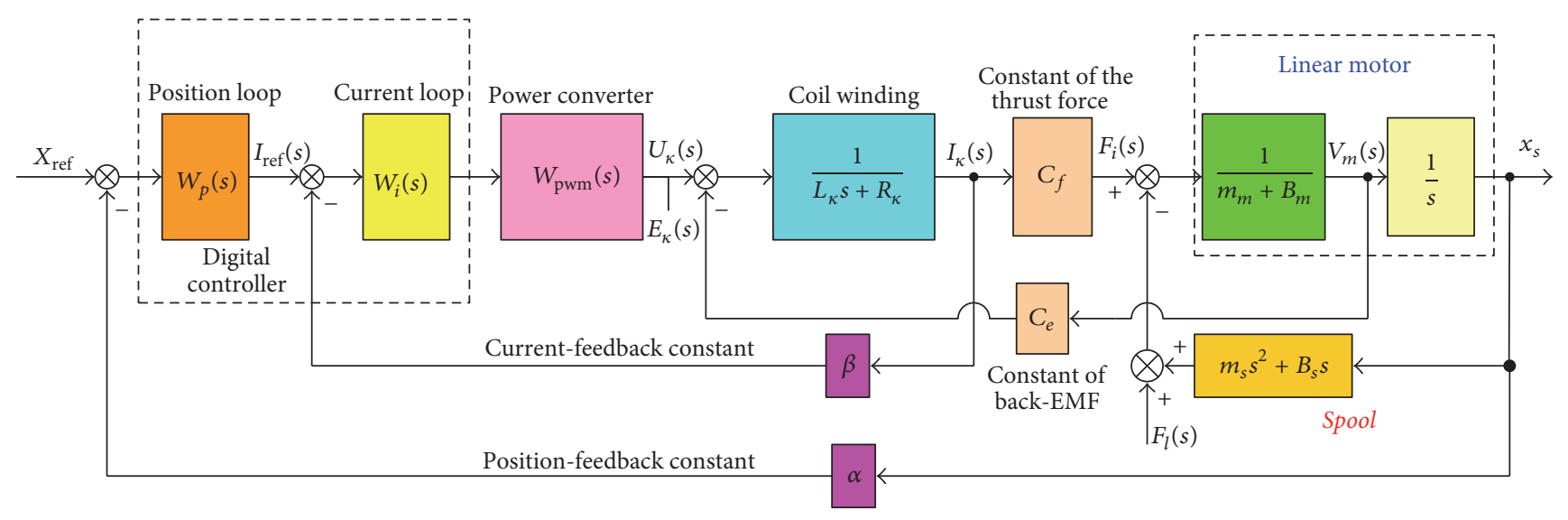

FIGURE 2: Block diagram of a double-loop intelligent controller. follows:

The high order model (order $=n$ ) can be expressed as

$$
\begin{gathered}
\dot{x}_{1}=x_{2}, \\
\dot{x}_{2}=x_{3}, \\
\vdots \\
\dot{x}_{n-1}=x_{n}, \\
\dot{x}_{n}=f\left(t, x_{1}, x_{2}, \ldots, x_{n}, w\right)+b u, \\
y=x_{1}+\Lambda,
\end{gathered}
$$

where $w$ is external disturbance variable, $u$ is control variable, $f\left(x, \dot{x}, \ldots, x^{(n-1)}, t\right)$ is an uncertain plant, and $\Lambda$ represents the output noise of the system.

The state-space model of the system is given in the following form:

$$
\begin{aligned}
& \dot{\mathbf{x}}(t)=A \mathbf{x}(t)+B(f(t)+u(t)), \\
& y(t)=C x(t)+\Lambda(t),
\end{aligned}
$$

where $A=\left[\begin{array}{ccccc}0 & 1 & \cdots & \cdots & 0 \\ 0 & 0 & 1 & \cdots & 0 \\ \vdots & \ddots & \ddots & \ddots & \vdots \\ 0 & \cdots & \cdots & 0 & 1 \\ 0 & 0 & \cdots & \cdots & 0\end{array}\right]_{n \times n} ; B=\left[\begin{array}{lllll}0 & 0 & \cdots & 1 & 0\end{array}\right]_{1 \times n}^{T} ; C=$ $\left[\begin{array}{lllll}1 & 0 & \cdots & \cdots & 0\end{array}\right]_{1 \times n}$.

Define $x_{n+1}=f(\cdot)$ as the extended state of the system described in (6), and define $\dot{\mathbf{x}}=\left[\begin{array}{lllll}x_{1} & x_{2} & \cdots & x_{n} & x_{n+1}\end{array}\right]^{T}$ as the whole state of the system.

So, the extended state observer should be described as

$$
\begin{gathered}
e=z_{1}-y, \\
z_{1}=\widehat{x}_{2}+\frac{\alpha_{1}}{\varepsilon}\left(x_{1}+\Lambda-\widehat{x}_{1}\right), \\
z_{2}=\widehat{x}_{3}+\frac{\alpha_{2}}{\varepsilon^{2}}\left(x_{1}+\Lambda-\widehat{x}_{1}\right), \\
\vdots \\
z_{n}=\widehat{x}_{n+1}+b_{0} u+\frac{\alpha_{n}}{\varepsilon^{n}}\left(x_{1}+\Lambda-\widehat{x}_{1}\right), \\
z_{n+1}=\frac{\alpha_{n+1}}{\varepsilon^{n+1}}\left(x_{1}+\Lambda-\hat{x}_{1}\right),
\end{gathered}
$$


where $\widehat{\mathbf{x}}=\left[\begin{array}{lllll}\hat{x}_{1} & \widehat{x}_{2} & \cdots & \widehat{x}_{n} & \widehat{x}_{n+1}\end{array}\right]^{T}$ is the observable of $\dot{\mathbf{x}}, e$ is the tracking error of the ESO, and $b_{0}$ is the estimated value of b. $\alpha_{1} \quad \alpha_{2} \cdots \alpha_{n} \alpha_{n+1}$ is the adjusting gain, and $\varepsilon$ is a small positive constant.

Define $\mathbf{M}^{T}=\left(\begin{array}{lllll}\alpha_{1} / \varepsilon & \alpha_{2} / \varepsilon^{2} & \cdots & \alpha_{n} / \varepsilon^{n} & \alpha_{n+1} / \varepsilon^{n+1}\end{array}\right)$ as the gain vector. In order to ensure the convergence of the ESO, the roots of polynomial (8) should all be located on the negative plane.

$$
s^{n+1}+\alpha_{1} s^{n}+\cdots+\alpha_{n} s+\alpha_{n+1}=0 .
$$

The existence of the output noise $\Lambda$ would have a bad influence on the performance of the ESO. When the gain of the ESO is larger, the tracking speed is faster but ESO would be more sensitive to the influence of the output noise $\Lambda$, so, in this paper, a switching rule is designed to tackle this problem.

According to the characteristics of the ESO, when the tracking error $e$ is large, a small parameter $\mathbf{M}_{1}^{T}$ is selected so as to estimate the system state and its uncertainty quickly. When the tracking error $e$ decreases to a certain value (meanwhile, ESO tracks the state and the extended state of the system), a large parameter $\mathbf{M}_{2}{ }^{T}$ is selected so that the system's sensitivity to the noise could be reduced and the high frequency of the noise could be filtered to some extent. $\mathbf{M}_{1}{ }^{T}$ and $\mathbf{M}_{2}{ }^{T}$ are

$$
\begin{aligned}
& \mathbf{M}_{1}^{T}=\left[\begin{array}{lllll}
\frac{\alpha_{11}}{\varepsilon} & \frac{\alpha_{12}}{\varepsilon^{2}} & \cdots & \frac{\alpha_{1 n}}{\varepsilon^{n}} & \frac{\alpha_{1(n+1)}}{\varepsilon^{n+1}}
\end{array}\right], \\
& \mathbf{M}_{2}^{T}=\left[\begin{array}{lllll}
\frac{\alpha_{21}}{\varepsilon} & \frac{\alpha_{22}}{\varepsilon^{2}} & \cdots & \frac{\alpha_{2 n}}{\varepsilon^{n}} & \frac{\alpha_{2(n+1)}}{\varepsilon^{n+1}}
\end{array}\right] .
\end{aligned}
$$

In order to avoid the duplicated switching of the proposed method, a delay timer is used. So, the procedure of the switching of the ESO is arranged as follows:

(1) When $|e|>\partial$, the delay timer is reset, and $\mathbf{M}_{1}{ }^{T}$ is selected.

(2) When $|e| \in[-\partial, \partial]$, the delay timer is activated, and $\mathbf{M}_{1}{ }^{T}$ is still selected.

(3) When the delay time is over, meanwhile $|e| \in[-\partial, \partial]$, and the gain of the ESO is switched to $\mathbf{M}_{2}{ }^{T}$.

Meanwhile, $\partial$ determines the switching region.

The position loop is most related to the dynamic and static performance of the DDA. In the proposed scheme, nonlinear arrangement is used to attenuate the influence of the hydraulic force. Tracking differentiator is arranged as follows:

$$
\begin{aligned}
& e=v_{1}-v_{0}, \\
& v_{1}=v_{1}+h \cdot v_{2}, \\
& v_{2}=v_{2}+h \cdot f_{\mathrm{st}}\left(e, v_{2}, r_{0}, h\right),
\end{aligned}
$$

where $f_{\mathrm{st}}\left(x_{1}, x_{2}, r, h\right)$ is the most comprehensive function, $v_{1}$ is the arranged transition of $v_{0}$, and $h$ is the sampling period.
The most comprehensive function $f_{\mathrm{st}}\left(x_{1}, x_{2}, r, h\right)$ is expressed as follows:

$$
\begin{aligned}
d & =\delta \cdot h, \\
d_{0} & =h \cdot d, \\
y & =x_{1}+h \cdot x_{2}, \\
a_{0} & =\sqrt{d^{2}+7 r \cdot|y|}, \\
a & = \begin{cases}x_{2}+\frac{\left(a_{0}-d\right)}{2} \operatorname{sign}(y), & |y|>d_{0} \\
x_{2}+\frac{y}{h}, & |y| \leq d_{0},\end{cases} \\
f_{\text {st }} & = \begin{cases}-\delta \cdot \operatorname{sign}(a), & |a|>d \\
\delta \frac{a}{d}, & |a| \leq d,\end{cases}
\end{aligned}
$$

where $\delta$ represents a coefficient which determines the tracking speed.

The nonlinear state feedback function is arranged as follows:

$$
\begin{aligned}
e_{0}(\tau)= & \int_{0}^{t} e_{1}(\tau) d \tau, \\
e_{1}= & v_{1}-z_{1}, \\
e_{2}= & v_{2}-z_{2}, \\
u= & \beta_{01} \cdot f a l\left(e_{0}, \alpha, \delta\right)+\beta_{02} \cdot f a l\left(e_{1}, \alpha, \delta\right)+\beta_{03} \\
& \cdot f a l\left(e_{2}, \alpha, \delta\right) .
\end{aligned}
$$

We propose the following nonlinear function:

$$
f a l(\varepsilon, \alpha, \delta)= \begin{cases}|\varepsilon|^{\alpha} \cdot \operatorname{sgn}(\varepsilon), & |\varepsilon|>\delta \\ \frac{\varepsilon}{\delta^{1-\alpha}}, & |\varepsilon| \leq \delta .\end{cases}
$$

RBF neural network has a three-layer feedforward network. It simulates the partial adjustment in the human brain and the receptive field. The activation functions of the neurons in the hidden layer are nonlinear functions. And the activation functions of the neurons in the output layer are linear functions. So, the learning speed could be accelerated and the local minimum problem could be avoided.

Three-layer RBF neural network's block diagram is designed in this paper, as shown in Figure 3.

In the structure of the RBF neural network, $\mathbf{X}=$ $\left[X_{1}, X_{2}, \ldots, X_{l}\right]$ is the input vector of the neural network, and the radial basis vector of the neural network is

$$
h_{j}=\exp \left(\frac{\left\|\mathbf{X}-\mathbf{c}_{j}\right\|}{2 d_{j}^{2}}\right), \quad j=1,2, \ldots, n,
$$

where $d_{j}$ represents the base width parameter and $\mathbf{c}_{j}=$ $\left[c_{j 1}, c_{j 2}, \ldots, c_{j l}\right]$ denotes the center vector of the $j$ th node in the hidden layer. The neural network output of the identification could be represented as follows:

$$
y_{n}(k)=w_{1} h_{1}+w_{2} h_{2}+\cdots+w_{n} h_{n} .
$$




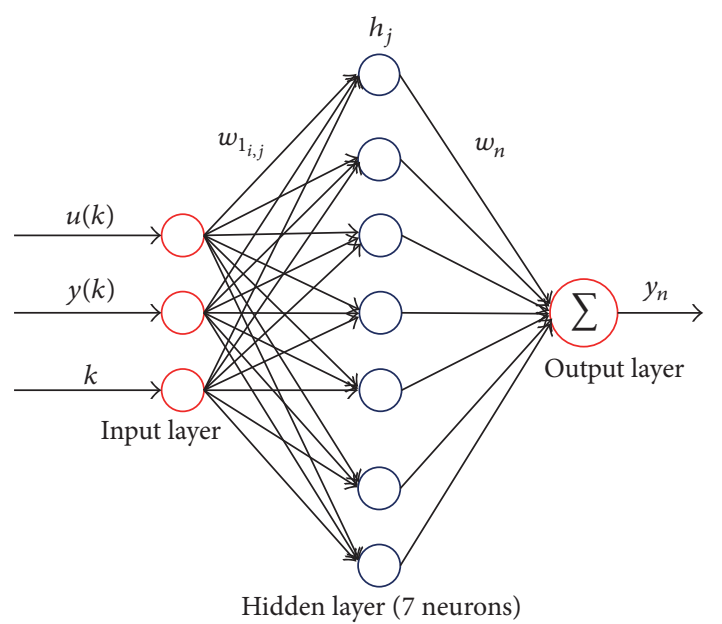

FIGURE 3: Block diagram of the RBF neural network optimization.

According to the principle of the gradient descent, the iteration algorithm to calculate the variation of the weight of the output layer, the center node, and the node based parameters could be expressed as

$$
\begin{aligned}
w_{j}(k)= & w_{j}(k-1)+\Delta w_{j}(k) \\
& +\alpha\left(w_{j}(k-1)-w_{j}(k-2)\right), \\
b_{j}(k)= & b_{j}(k-1)+\Delta b_{j} \\
& +\alpha\left(b_{j}(k-1)-b_{j}(k-2)\right), \\
c_{j i}(k)= & c_{j i}(k-1)+\Delta c_{j i}(k) \\
& +\alpha\left(c_{j i}(k-1)-c_{j i}(k-2)\right), \\
\Delta w_{j}(k)= & \eta\left(y(k)-y_{n}(k)\right) h_{j}, \\
\Delta d_{j}(k)= & \eta\left(y(k)-y_{n}(k)\right) \frac{\left\|\mathbf{X}-\mathbf{c}_{j}\right\|^{2}}{2 d_{j}^{2}}, \\
\Delta c_{j l}(k)= & \eta\left(y(k)-y_{n}(k)\right) w_{j} \frac{X_{j}-c_{j i}}{d_{j}^{2}},
\end{aligned}
$$

where $\alpha$ is the momentum factor and $\eta$ is the learning rate. The setting indicator of the neural network is expressed as

$$
E(k)=\frac{1}{2}(v(k)-y(k))^{2},
$$

where $v(k)$ and $y(k)$ are position command signal and the position response signal.
TABLE 1: Major specification data.

\begin{tabular}{lc}
\hline Parameters & Quantity \\
\hline Voltage $(\mathrm{V})$ & 48.2 \\
Maximum current $(\mathrm{A})$ & 6.5 \\
Inductance $(\mathrm{mH})$ & 2.3 \\
Resistor $(\Omega)$ & 5.1 \\
Coefficient of the force $(\mathrm{N} / \mathrm{A})$ & 24 \\
Frequency of the PWM $(\mathrm{Hz})$ & 50 \\
Full stroke (mm) & \pm 1.2 \\
Rated thrust force of the motor $(\mathrm{N})$ & 79 \\
Quality of the moving coil $(\mathrm{kg})$ & 0.11 \\
Hydraulic pressure $(\mathrm{MPa})$ & 25 \\
\hline
\end{tabular}

The adjustment of $\beta_{01}, \beta_{02}$, and $\beta_{03}$ utilizes the gradient descent method, which could be represented as

$$
\begin{aligned}
& \Delta \beta_{01}=-\eta \frac{\partial E}{\partial \beta_{01}}=-\eta \frac{\partial E}{\partial y} \frac{\partial y}{\partial \Delta u} \frac{\partial \Delta u}{\partial \beta_{01}} \\
& \Delta \beta_{02}=-\eta \frac{\partial E}{\partial \beta_{02}}=-\eta \frac{\partial E}{\partial y} \frac{\partial y}{\partial \Delta u} \frac{\partial \Delta u}{\partial \beta_{02}} \\
& \Delta \beta_{03}=-\eta \frac{\partial E}{\partial \beta_{03}}=-\eta \frac{\partial E}{\partial y} \frac{\partial y}{\partial \Delta u} \frac{\partial \Delta u}{\partial \beta_{03}}
\end{aligned}
$$

The diagram of the control algorithm (RBF-ESO) proposed in this paper is shown in Figure 4.

\section{Experiments}

To confirm the precision and practicability of the proposed control method for the LM-DDA system, the system was first built using MATLAB/Simulink. The simulation experiment was done on the PID control, the RBF neural network PID control, and the proposed algorithm in Section 3 on the LMDDA system. The results of the PID control, the RBF neural network PID control (RBF-PID), and the RBF neural network control based on the extended state observer (RBF-ESO) were compared.

We choose $\eta=0.015$ and $\alpha=0.85$ for supervised learning. Figure 5 shows the learning curve of the RBF model. This figure denotes the mean squared error (MSE) along the training step. From the figure, we could see that the convergence effects could be reached.

The major specification data are listed in Table 1.

It is clearly seen in Figure 6 that the settling process of the system is more time-consuming $(32.3 \mathrm{~ms})$ while utilizing classic PID control strategy. While applying the RBF-PID, the overshoot is weakened and the system's settling time is shortened by comparison to the PID control. In contrast, the settling time is the shortest $(18.1 \mathrm{~ms})$ when the proposed control strategy (RBF-ESO) is applied. And the DDA system almost has no overshoot and vibration while applying the algorithm.

There are such uncertainties that the mechanical characteristics might change when the actuator is running. Figures $7(\mathrm{a})-7$ (c) show the command and the positon response of the DDA system when the quality shifts during the working 


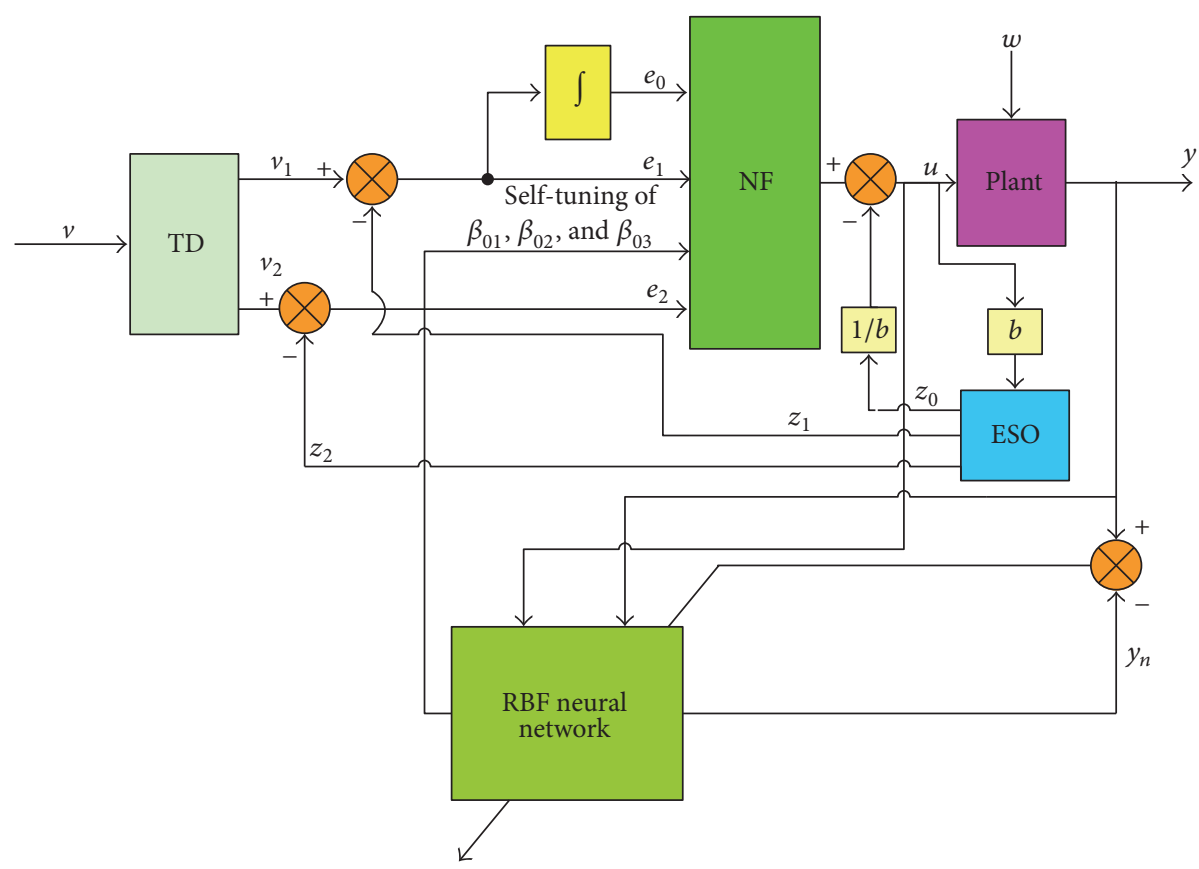

FIgURE 4: Control diagram of the RBF-ESO algorithm proposed in this paper.

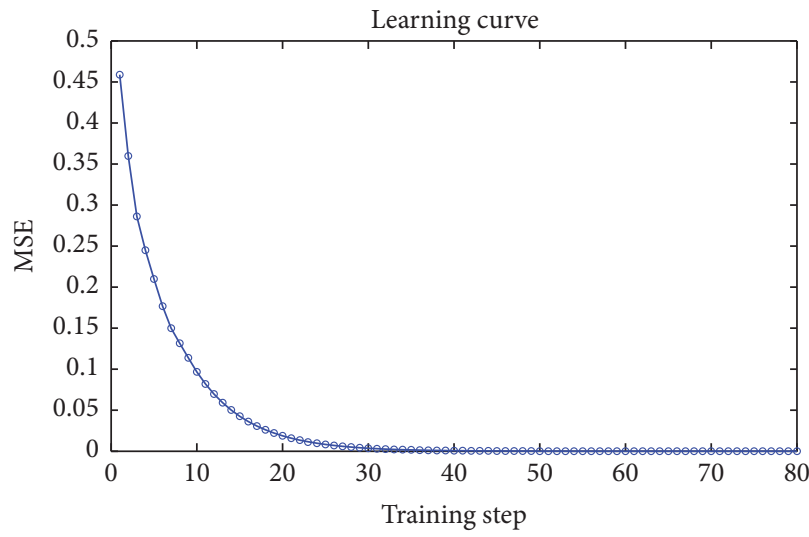

FIgURE 5: The learning curve of the RBF neural network.

process $\left(m_{m}=0.11 \mathrm{~kg}\right)$. The figures validate the notion that the RBF-ESO control scheme shows the best robustness. Compared to the PID control method and the RBF-PID control method, the trajectory curve shows only little difference when the quantity doubles.

Figure 8 shows the system's hardware structure based on the TMS28335 DSP and EP2C35F672C6 FPGA. And the experimental setup is shown in Figure 9.

There are two loops in the LM-DDA control system: the position loop and the current loop. The calculation of the position loop is accomplished by the DSP and the calculation of the current loop is realized in the FPGA. The proposed control strategy is fulfilled in the position loop. Thus, the DSP would be concentrated on the calculation of the proposed algorithm.
A simulation testing VHDL code after compiling and generating the configuration file is done and then the data is downloaded to the FPGA. Since the linear motor is used for coreless motors, the winding inductance is small, thus increasing the difficulty of current control. In this case, a short cycle $(20 \mu \mathrm{s})$ of the current loop calculation is used in the system in order to achieve the good effect of the current control. The current loop controller uses the configuration of FPGA, which would have a simple structure, control speed, and high reliability.

A displacement sensor is set up to get the position feedback signal. Then, this signal is transmitted to the FPGA and then is transmitted to the DSP. The position reference sent from DSP is sent to the FPGA through the sequential port. The power drive circuit is connected directly to the LM, 


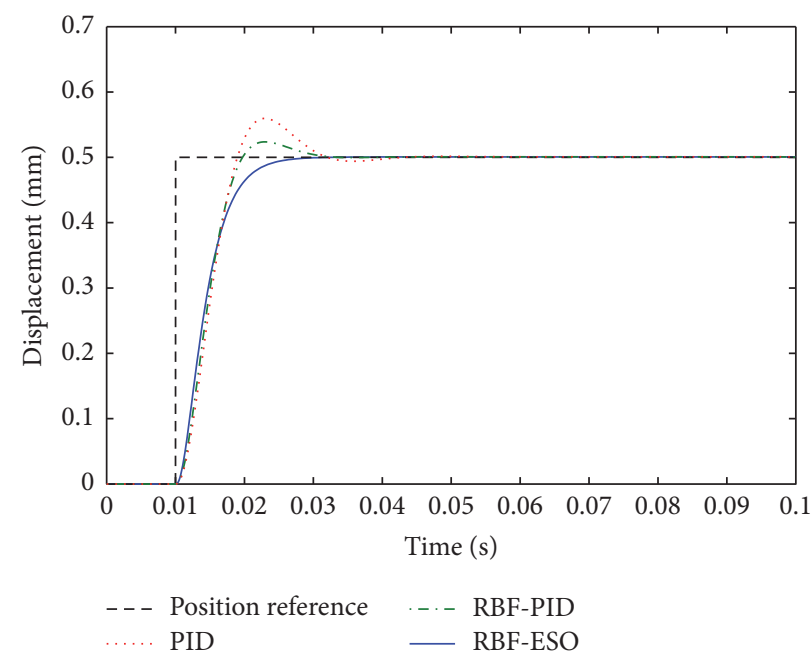

FIGURE 6: Tracking of position step response by different algorithms.

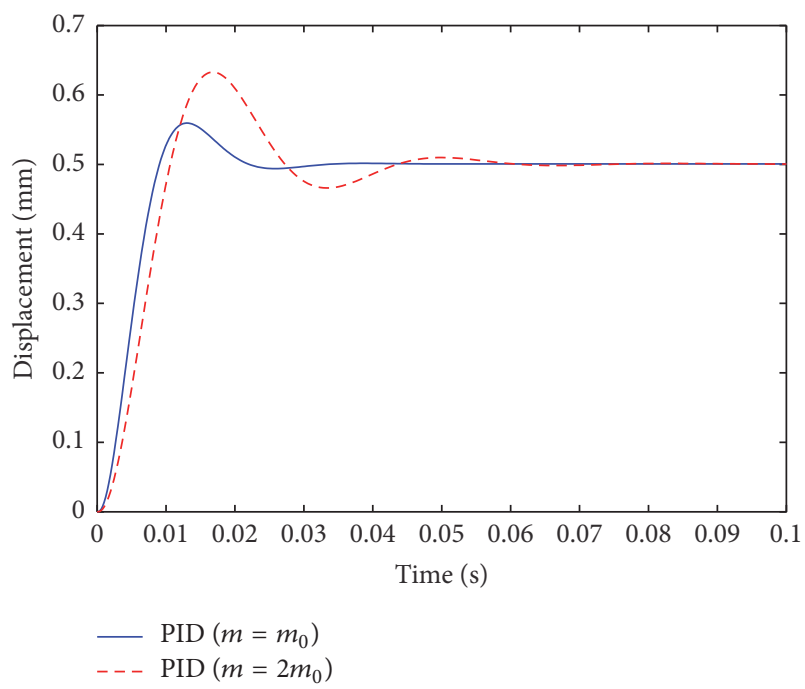

(a)

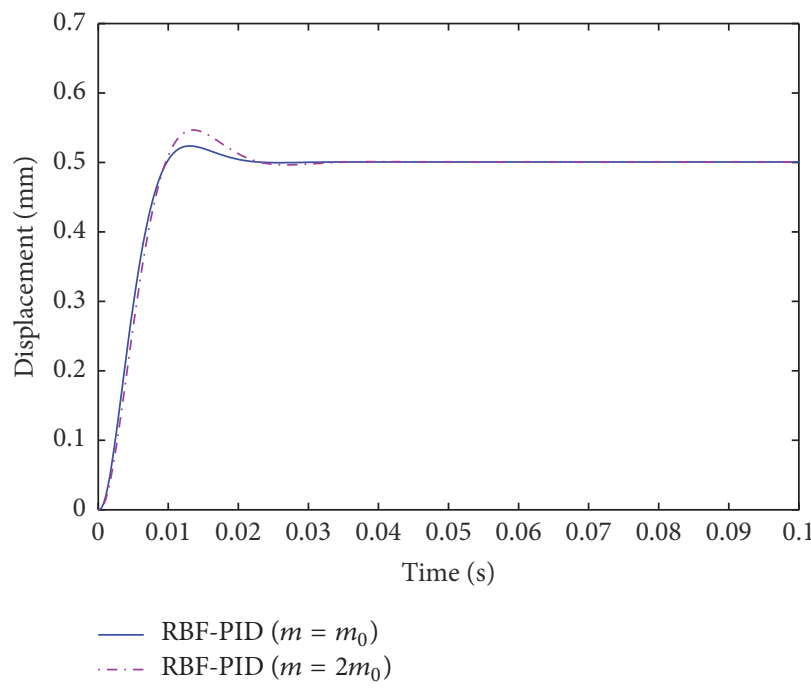

(b)

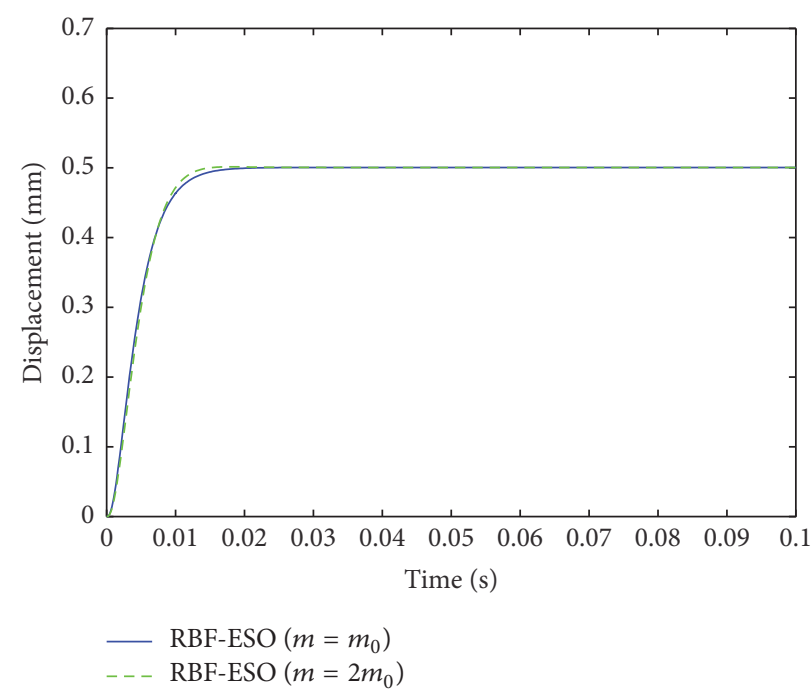

(c)

FiguRE 7: Robustness of different algorithms to diversification of the mechanical characteristic. 


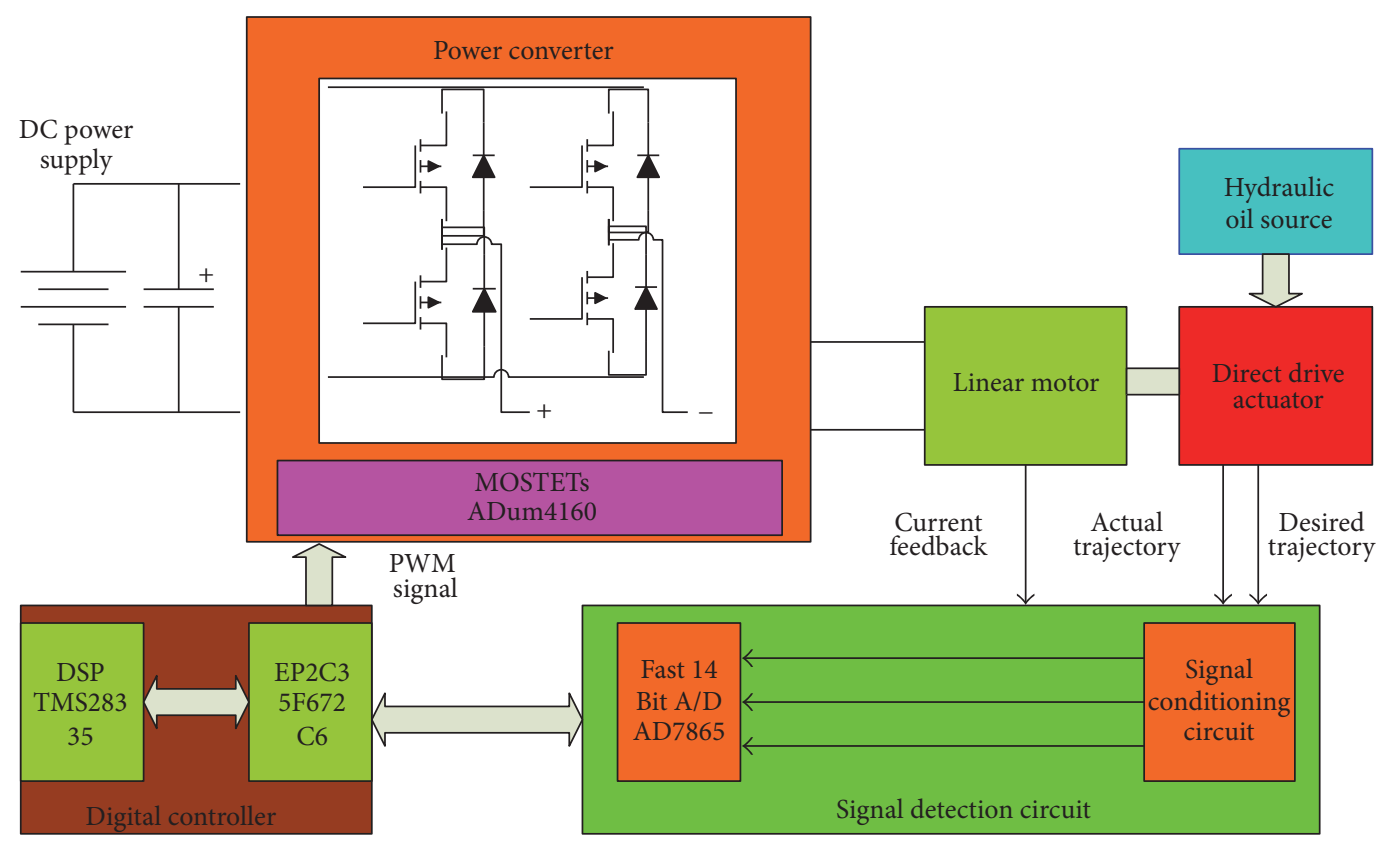

FIGURE 8: Schematic of system implementation.

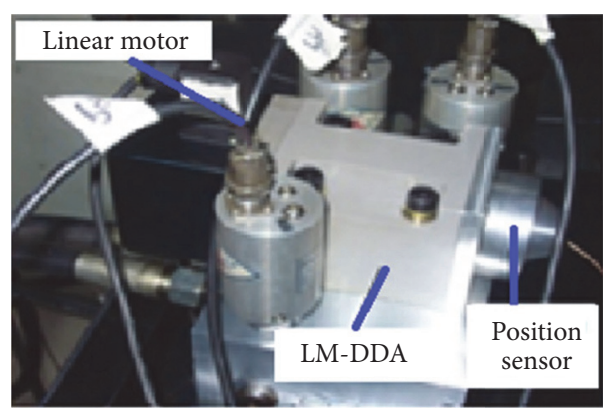

FIGURE 9: Experimental setup of the LM-DDA system.

and it receives the PWM signal generated by the FPGA and transforms it into 2-bridge power gate. The power circuit is composed of two parts: the isolated circuit and the full bridge inverter circuit. And the overcurrent fortification function is also realized by the FPGA. The experiments of the DDA system are accomplished according to Figures 10 and 11.

Figure 10(a) shows the results of the tracking waveform of the PID control and the proposed scheme. Fluid power and other disturbances on the system would have great influence on the tracking performance while using the PID control. As is shown in Figure 10(b), the LM-DDA system could attenuate the influence of the fluid power and other load disturbances while using the RBF-PID control but the system still has overshoot and vibration due to the unpredictable hydraulic force. From Figure 10(c), we could see that the position tracking is fast and there is almost no overshoot while applying the strategy of RBF-ESO.

Figure 11 shows the LM-DDA waveforms while tracking the $100 \mathrm{~Hz}$ sine wave when the proposed control strategy (RBF-ESO) is employed. It shows the rapid response of the system while applying the proposed RBF-ESO control method.

The experimental results on the LM-DDA system indicate that the strategy proposed can effectively overcome the influence of fluid power load on the system performance. Despite the influence of the fluid power and load disturbance, the system can maintain good stability and dynamic performance and would meet the system requirements.

\section{Conclusions}

The load disturbances in a linear motor-direct drive actuator (LM-DDA) have great influence on the system performance. A mathematical model of the LM-DDA system is established and a double-loop control system is presented. An extended state observer (ESO) with switched gain was utilized to estimate the influence of the hydraulic power and other load disturbances. Meanwhile, RBF neural network was utilized to optimize the parameters in this system. Simulation and experimental results demonstrated that the performance with 


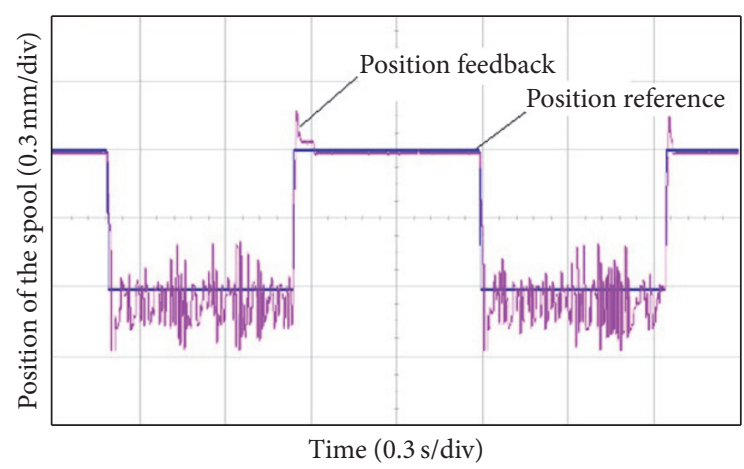

(a)

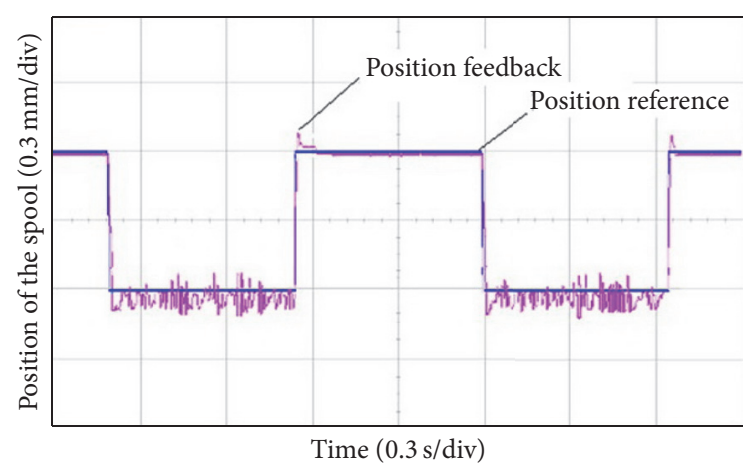

(b)

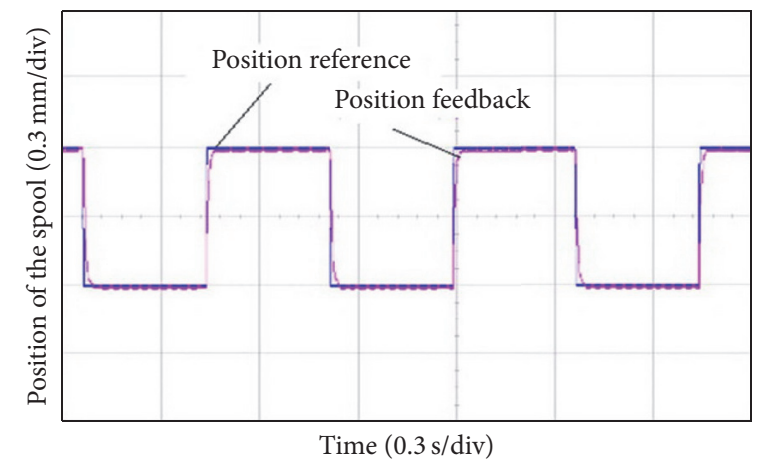

(c)

FIGURE 10: Experimental results of square trajectory tracking (a) using conventional PID control, (b) using RBF-PID method, and (c) using RBF-ESO method.

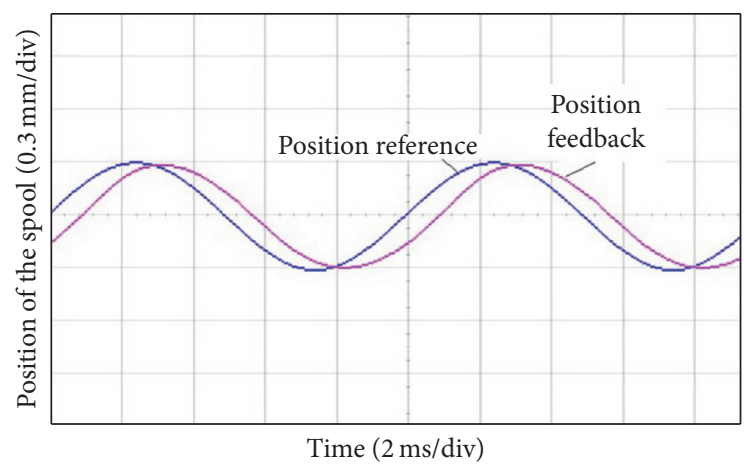

FIGURE 11: Experimental results of the $100 \mathrm{~Hz}$ sinusoid trajectory tracking using RBF-ESO method.

rapid response and improved accuracy could be achieved by the proposed control scheme.

\section{Competing Interests}

The authors declare that there are no competing interests regarding the publication of this paper.

\section{Acknowledgments}

This work was supported by the National Natural Science Foundation of China under Grant no. 61273158.

\section{References}

[1] S. J. Fu, M. Liffring, and I. S. Mehdi, "Integrated electro-hydraulic system modeling and analysis," IEEE Aerospace and Electronic Systems Magazine, vol. 17, no. 7, pp. 4-8, 2002.

[2] Z. Yang, Z. He, D. Li, J. Yu, X. Cui, and Z. Zhao, "Direct drive servo valve based on magnetostrictive actuator: multicoupled modeling and its compound control strategy," Sensors and Actuators, A: Physical, vol. 235, pp. 119-130, 2015.

[3] B. Na, H. Choi, and K. Kong, "Design of a direct-driven linear actuator for a high-speed quadruped robot, cheetaroidI," IEEE/ASME Transactions on Mechatronics, vol. 20, no. 2, pp. 924-933, 2015.

[4] J. Jeon, C. Han, Y. M. Han, and S. B. Choi, "A new type of a direct-drive valve system driven by a piezostack actuator and sliding spool," Smart Materials and Structures, vol. 23, no. 7, Article ID 075002, 13 pages, 2014.

[5] H. S. Liu, Y. L. Fu, J. Chen, and H. Chen, "Active disturbance rejection control in airborne direct drive electro mechanical actuator application," in Proceedings of the International Conference on Design, Manufacturing and Mechatronics (ICDMM '14), pp. 541-547, January 2014.

[6] A. Z. Shukor and Y. Fujimoto, "Direct-drive position control of a spiral motor as a monoarticular actuator," IEEE Transactions on Industrial Electronics, vol. 61, no. 2, pp. 1063-1071, 2014.

[7] P. A. Toensmeier, "Direct-drive motor and nonlinear actuator boost productivity of electric injection unit," Plastics Engineering, vol. 63, no. 4, pp. 38-40, 2007.

[8] H.-T. Yau, Y.-C. Pu, and S. C. Li, "An FPGA-based PID controller design for chaos synchronization by evolutionary 
programming," Discrete Dynamics in Nature and Society, vol. 2011, Article ID 516031, 11 pages, 2011.

[9] M. C. Razali, N. A. Wahad, P. Balaguer, M. F. Rahmat, and S. I. Samsudin, "Singularly perturbation method applied to multivariable PID controller design," Mathematical Problems in Engineering, vol. 2015, Article ID 818353, 22 pages, 2015.

[10] K. H. Ang, G. Chong, and Y. Li, "PID control system analysis, design, and technology," IEEE Transactions on Control Systems Technology, vol. 13, no. 4, pp. 559-576, 2005.

[11] A. Farag and H. Werner, "Structure selection and tuning of multi-variable PID controllers for an industrial benchmark problem," IEE Proceedings: Control Theory and Applications, vol. 153, no. 3, pp. 262-267, 2006.

[12] Y. Wu and J. Wang, "Continuous recursive sliding mode control for hypersonic flight vehicle with extended disturbance observer," Mathematical Problems in Engineering, vol. 2015, Article ID 506906, 26 pages, 2015.

[13] L. Zhao and Y. M. Jia, "Neural network-based adaptive consensus tracking control for multi-agent systems under actuator faults," International Journal of Systems Science, vol. 47, no. 8, pp. 1931-1942, 2016.

[14] L. Zhang, K. Li, and E.-W. Bai, "A new extension of Newton algorithm for nonlinear system modelling using RBF neural networks," IEEE Transactions on Automatic Control, vol. 58, no. 11, pp. 2929-2933, 2013.

[15] Z. Gao, "Active disturbance rejection control for nonlinear fractional-order systems," International Journal of Robust and Nonlinear Control, vol. 26, no. 4, pp. 876-892, 2016.

[16] H. Li, X. Q. Liu, and J. Li, “The research of Fuzzy Immune Linear Active Disturbance Rejection Control Strategy for threemotor synchronous system," Control Engineering and Applied Informatics, vol. 17, no. 4, pp. 50-58, 2015.

[17] Z.-L. Zhao and B.-Z. Guo, "Extended state observer for uncertain lower triangular nonlinear systems," Systems and Control Letters, vol. 85, pp. 100-108, 2015. 


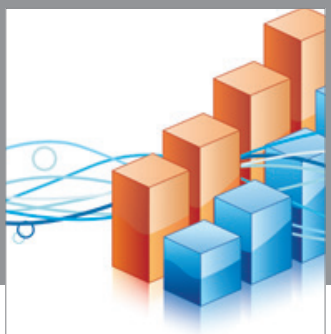

Advances in

Operations Research

vatem alat4

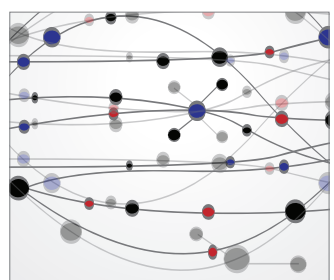

\section{The Scientific} World Journal
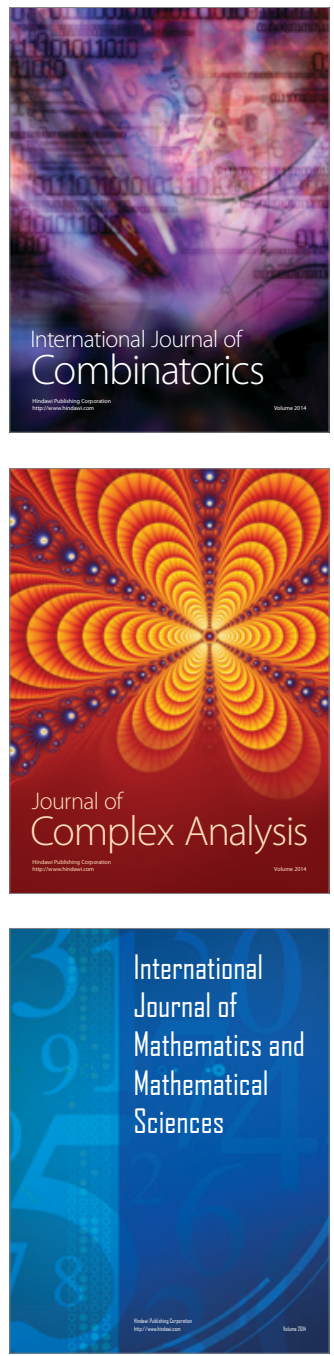
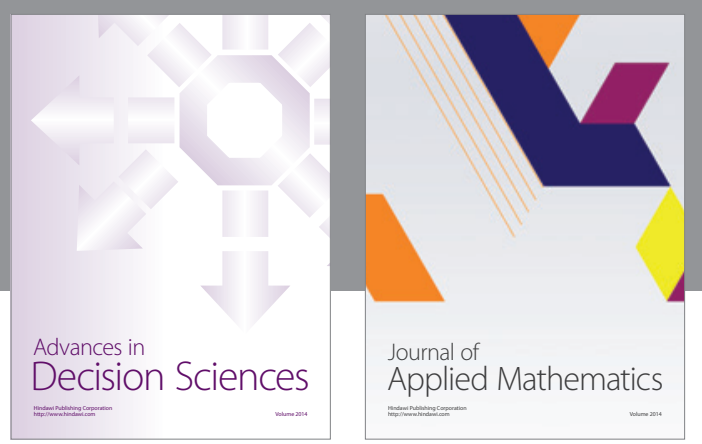

Algebra

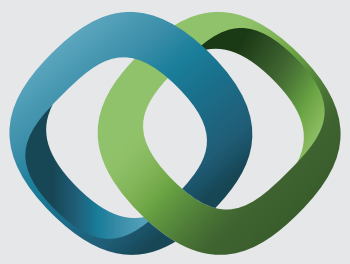

\section{Hindawi}

Submit your manuscripts at

http://www.hindawi.com
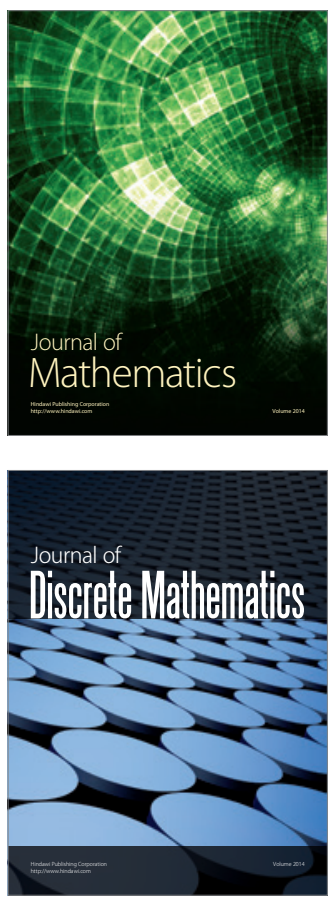

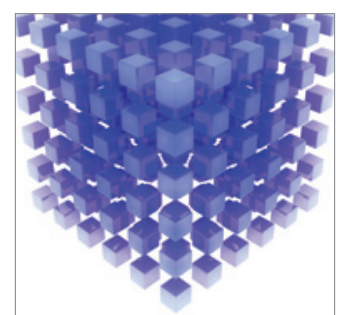

Mathematical Problems in Engineering
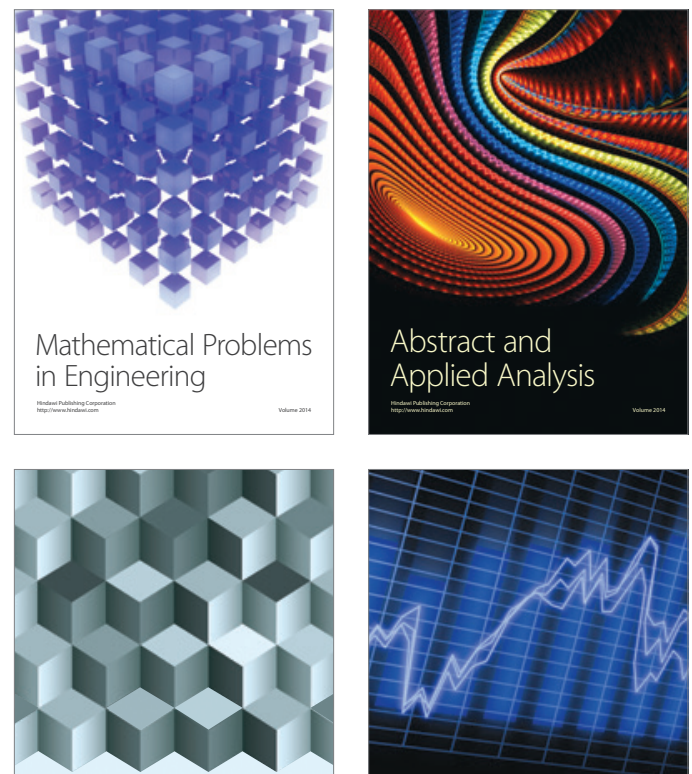

Journal of

Function Spaces

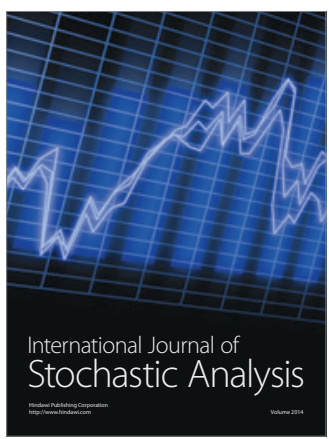

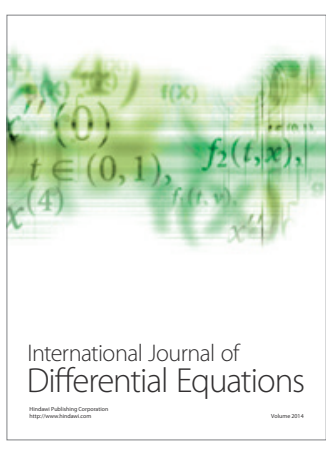
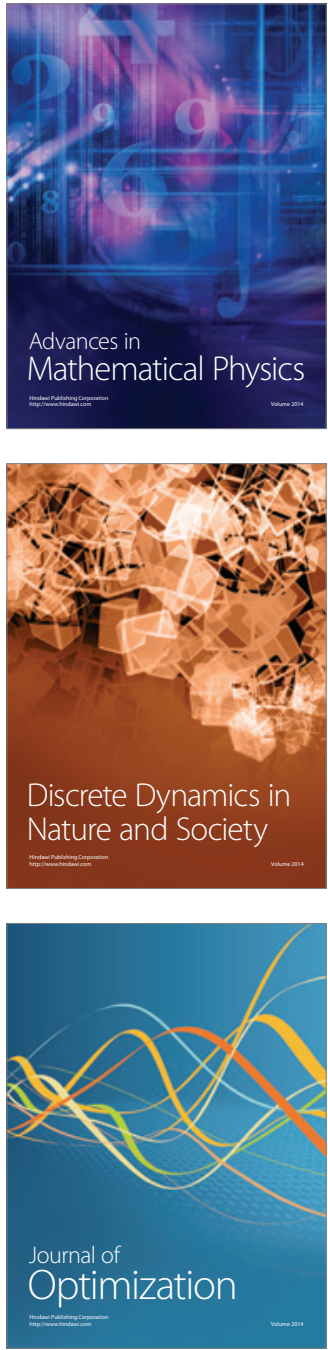